\title{
A CLASS OF SINGULAR NEUTRAL-DIFFERENTIAL SYSTEMS ${ }^{1}$
}

\author{
W. J. FITZPATRICK AND L. J. GRIMM
}

\begin{abstract}
Noetherian operator theory is used to prove an existence theorem for a singular functional-differential system. An analogue of the standard existence and uniqueness results at an ordinary point follows as a corollary.
\end{abstract}

Functional-differential equations of the form

$$
y^{\prime}(z)=A(z) y(z)+B(z) y(\alpha z)
$$

have been studied by T. Kato and J. B. McLeod [6] and others. Recently Ll. G. Chambers [1] has obtained sufficient conditions for existence of holomorphic solutions of the scalar equation with deviating arguments and constant coefficients of the form

$$
y^{\prime}(z)=\sum_{n=0}^{\infty} a_{n} y\left(\mu^{n} z\right),
$$

where $\mu$ is a constant with $|\mu|<1$, and V. P. Skripnik [8] has studied stability of a corresponding nonlinear equation.

A singular equation analogous to (1) has also received some attention, particularly in the Soviet literature; see, for example, the references in [2] or [4]. We obtain here existence results for a singular neutral-differential system with deviating arguments similar to (2). The principal result is a generalization of the classical Lettenmeyer theorem [5].

Let $B_{1}$ and $B_{2}$ be Banach spaces and let $T: B_{1} \rightarrow B_{2}$ be a continuous linear operator with domain $B_{1}$. Denote the conjugate of $T$ by $T^{*}$.

Definition 1. The operator $T$ is called normally solvable if the set $T\left(B_{1}\right)$ is closed in $B_{2}$.

Definition 2. The $d$-characteristic of the operator $T$ is the ordered pair $(\alpha(T), \beta(T))$, where $\alpha(T)=\operatorname{dim}(\operatorname{ker} T)$ and $\beta(T)=\operatorname{dim}\left(B_{2} / T\left(B_{1}\right)\right)$.

Definition 3. The index of the operator $T$ is the number $\beta(T)-\alpha(T)$, denoted by ind $(T)$.

Definition 4. The operator $T$ is called a Noetherian operator if $T$ is normally solvable and has a finite $d$-characteristic, i.e., both $\alpha(T)$ and $\beta(T)$ are finite.

Received by the editors June 28, 1976 and, in revised form, November 10, 1976.

AMS (MOS) subject classifications (1970). Primary 34A20, 34K05.

Key words and phrases. Singular points, neutral-differential equations, holomorphic solutions.

${ }^{1}$ Research supported by NSF Grant MCS 76-08229. 
REMARK. If $\beta(T)<\infty$, then $\beta(T)=\operatorname{dim}\left(\operatorname{ker} T^{*}\right)$.

The following theorem is due to I. C. Gohberg and M. G. Krein [3].

THEOREM 1. Let T: $B_{1} \rightarrow B_{2}$ be a continuous Noetherian operator, and let $T_{1}$ : $B_{1} \rightarrow B_{2}$ be a compact operator. Then $T_{2}=T+T_{1}$ is a Noetherian operator from $B_{1}$ into $B_{2}$ with $\operatorname{ind}\left(T_{2}\right)=\operatorname{ind}(T)$.

Let $r>0$, let $G_{r}=\{z:|z|<r\}$ and define $A_{p}\left(G_{r}\right)$ to be the Banach space of functions $v(z)$ holomorphic in $G_{r}$ and $p$ times continuously differentiable on $\bar{G}_{r}$ with norm

$$
\|v(z)\|_{p}=\max _{z \in \bar{G}_{r}}\left|v^{(i)}(z)\right|, \quad 0 \leqslant i \leqslant p .
$$

Let $A_{p, n}\left(G_{r}\right)$ be the Banach space of $n$-vector functions $y(z)=$ $\left(y^{1}(z), \ldots, y^{n}(z)\right)^{T}$, where $y^{k}(z) \in A_{p}\left(G_{r}\right), 1 \leqslant k \leqslant n$, with norm

$$
\|y(z)\|_{p}^{n}=\max \left\|y^{k}(z)\right\|_{p}, \quad 1 \leqslant k \leqslant n .
$$

For a matrix $A(z)=\left(a_{i j}(z)\right)$, with $a_{i j}(z) \in A_{0}\left(G_{r}\right)$, define

$$
\|A(z)\|=\max \left\|a_{i j}(z)\right\|_{0}, \quad 1 \leqslant i, j \leqslant n .
$$

Note that $\|A(z) y(z)\|_{0}^{n}=\|A(z)\|\|y(z)\|_{0}^{n}$.

Consider the differential equation

$$
A(z) y^{\prime}(z)+\sum_{i=1}^{\infty} B_{i}(z) y\left(\alpha_{i} z\right)+\sum_{i=1}^{\infty} C_{i}(z) y^{\prime}\left(\beta_{i} z\right)=0,
$$

where $A(z), B_{i}(z), C_{i}(z)$ are $n \times n$ matrices with elements in $A_{0}\left(G_{r}\right)$, $\sum_{i=1}^{\infty}\left\|B_{i}(z)\right\|<\infty, \sum_{i=1}^{\infty}\left\|C_{i}(z)\right\|<\infty$, and $\left\{\alpha_{i}\right\}$ and $\left\{\beta_{i}\right\}$ are sequences of complex constants with $0<\left|\alpha_{i}\right| \leqslant 1,0<\left|\beta_{i}\right|<1$.

THEOREM 2. Let $s$ be the number of zeros, counted algebraically, of det $A(z)$ in $G_{r}$. Then the system (3) has at least $n-s$ linearly independent solutions holomorphic in $G_{r}$.

Proof. Consider the operators $l^{(1)}$ and $l^{(2)}$ defined by

$$
\begin{array}{ll}
l^{(1)} y(z)=B(z) y(\alpha z), & |\alpha| \leqslant 1, \\
l^{(2)} y(z)=C(z) y^{\prime}(\beta z), & |\beta|<1 ;
\end{array}
$$

$l^{(1)}$ and $l^{(2)}$ each map $A_{1, n}\left(G_{r}\right)$ into $A_{0, n}\left(G_{r}\right)$. Let $Q$ be the family of $n$-vectors $h(z) \in A_{1}, n\left(G_{r}\right)$ such that $\|h\|_{1}^{n} \leqslant C<\infty$ for all $h \in Q$. Let $\hat{h}(z)=h(\alpha z)$. For all $h(z)=\left(h_{1}(z), \ldots, h_{n}(z)\right) \in Q$, each $j=1, \ldots, n$, and $z \in \bar{G}_{r}$,

$$
\begin{aligned}
& \left|\hat{h_{j}}(z)\right|=\left|h_{j}(\alpha z)\right| \leqslant \sup _{z \in \bar{G}_{r}}\left|h_{j}(z)\right| \leqslant C \text { and } \\
& \left|\hat{h}_{j}^{\prime}(z)\right|=\left|\alpha h_{j}^{\prime}(\alpha z)\right| \leqslant \sup _{z \in \bar{G}_{r}}\left|h_{j}^{\prime}(z)\right| \leqslant C,
\end{aligned}
$$

by the maximum modulus theorem. Hence the family $\left\{\hat{h}_{j}(z)\right\}_{h \in Q}$ is uniformly bounded and equicontinous on $\bar{G}_{r}$. From Ascoli's lemma, it is easy to see that 
$\{\hat{h}(z)\}_{h \in Q}$ contains a sequence convergent in $A_{0, n}\left(G_{r}\right)$. Thus $l^{(1)}: A_{1, n}\left(G_{r}\right) \rightarrow$ $A_{0, n}\left(G_{r}\right)$ is a compact operator.

Now set $\tilde{h}(z)=h(\beta z)$. By Montel's theorem, $\left\{h_{j}^{\prime}(z)\right\}_{h \in Q}$ is a normal family for $|z|<r$, hence $\left\{\tilde{h}_{j}^{\prime}(z)\right\}_{h \in Q}$ is a normal family for $|z|<r /|\beta|$. This means that $\left\{\tilde{h}_{j}^{\prime}(z)\right\}_{h \in Q}$ contains a sequence almost uniformly convergent on $|z|<r /|\beta| .\{z:|z| \leqslant r\}$ is a compact subset of $\{z:|z|<r /|\beta|\}$, so $\left\{\tilde{h}^{\prime}(z)\right\}_{z \in Q}$ clearly contains a sequence convergent in $A_{0, n}\left(G_{r}\right)$. Thus $l^{(2)}$ : $A_{1, n}\left(G_{r}\right) \rightarrow A_{0, n}\left(G_{r}\right)$ is a compact operator.

We have now shown that the operators $L_{k}^{(1)}, L_{k}^{(2)}: A_{1, n}\left(G_{r}\right) \rightarrow A_{0, n}\left(G_{r}\right)$ defined by

$$
\begin{aligned}
& L_{k}^{(1)} y(z)=\sum_{i=1}^{k} B_{i}(z) y\left(\alpha_{i} z\right) \quad \text { and } \\
& L_{k}^{(2)} y(z)=\sum_{i=1}^{k} C_{i}(z) y^{\prime}\left(\beta_{i} z\right)
\end{aligned}
$$

are compact.

Now consider

$$
\begin{gathered}
L^{(1)} y(z)=\sum_{i=1}^{\infty} B_{i}(z) y\left(\alpha_{i} z\right) \text { and } L^{(2)} y(z)=\sum_{i=1}^{\infty} C_{i}(z) y^{\prime}\left(\beta_{i} z\right) . \\
\left\|L^{(1)} y(z)\right\|_{0}^{n} \leqslant \sum_{i=1}^{\infty}\left\|B_{i}(z)\right\|\left\|y\left(\alpha_{i} z\right)\right\|_{0}^{n} \leqslant \sum_{i=1}^{\infty}\left\|B_{i}(z)\right\|\|y(z)\|_{1}^{n} .
\end{gathered}
$$

Since $\sum_{i=1}^{\infty}\left\|B_{i}(z)\right\|<\infty, L_{k}^{(1)} \rightarrow L^{(1)}$ uniformly in $A_{1, n}\left(G_{r}\right) .\left\{L_{k}^{(1)}\right\}$ is a sequence of compact operators, hence $L^{(1)}$ is compact. Similarly, $L^{(2)}$ is compact.

Korobeinik [7] has shown that the operator $L^{(0)}: A_{1, n}\left(G_{r}\right) \rightarrow A_{0, n}\left(G_{r}\right)$ defined by $L^{(0)} y(z)=A(z) y^{\prime}(z)$ is Noetherian with index $s-n$, where $s$ is the number of zeros, counted algebraically, of $\operatorname{det} A(z)$ in $G_{r}$. By Theorem 1, the operator $L=L^{(0)}+L^{(1)}+L^{(2)}$ is Noetherian with index $s-n$. But $\beta(L) \geqslant$ 0 , and thus $\operatorname{dim}(\operatorname{ker} L)=\alpha \geqslant n-s$. This completes the proof.

If we assume that $A(0)$ is nonsingular, we obtain the following corollary, which yields an analogue of the standard existence results at an ordinary point, from simple uniqueness considerations together with the fact that the space of functions holomorphic at zero can be represented as the limit of the spaces $A_{1}\left(G_{r}\right)$ as $r$ tends to zero:

COROLlaRY. With notation as in (2), the differential equation

$$
A(z) y^{\prime}(z)=\sum_{i=1}^{\infty} B_{i}(z) y\left(\alpha_{i} z\right)+\sum_{i=1}^{\infty} C_{i}(z) y^{\prime}\left(\beta_{i} z\right)
$$

has exactly $n$ linearly independent solutions holomorphic at $z=0$, provided that $A(0)$ is nonsingular.

\section{REFERENCES}

1. Ll. G. Chambers, Some functional differential equations, Quart. Appl. Math. 32 (1975), 445-456. 
2. W. J. Fitzpatrick, L. J. Grimm, and L. M. Hall, Singular point theory and a class of complex functional differential equations, Proc. Fifth All-Union Conf. on Functional Differential Equations, Kiev 1976 (to appear).

3. I. C. Gohberg and M. G. Krein, The basic propositions on defect numbers, root numbers, and indices of linear operators, Uspehi Mat. Nauk 12 (1957), 43-118; English transl., Amer. Math. Soc. Transl. (2) 13 (1960), 185-264. MR 20 \#3459; 22 \#3984.

4. L. J. Grimm and L. M. Hall, Holomorphic solutions of functional differential systems near singular points, Proc. Amer. Math. Soc. 42 (1974), 167-170. MR 48 \#6604.

5. W. A. Harris, Jr., Y. Sibuya and L. Weinberg, Holomorphic solutions of linear differential systems at singular points, Arch. Rational Mech. Anal. 35 (1969), 245-248. MR 40 \#432.

6. T. Kato and J. B. McLeod, The functional-differential equation $y^{\prime}(x)=a y(\lambda x)+b y(x)$, Bull. Amer. Math. Soc. 77 (1971), 891-937.

7. Ju. F. Korobeinik, Normal solvability of linear differential equations in the complex plane, Izv. Akad. Nauk SSSR Ser. Mat. 36 (1972), 450-474 = Math. USSR Izv. 6 (1972), 445-466. MR 46 \#724.

8. V. P. Skripnik, Stability of systems with a countable number of transformations of the argument, Differencial'nye Uravnenija 11 (1975), 841-849, 948. MR 52 \#963.

Department of Mathematics, University of Missouri, Rolla, Missouri 65401 (Curtent address of L. J. Grimm)

Current address (W. J. Fitzpatrick): Department of Mathematics, University of Southern California, Los Angeles, California 90007 